\title{
EVALUATING PERCEPTUALLY PREFILTERED VIDEO
}

\section{Olivier Steiger, Touradj Ebrahimi}

\author{
Ecole Polytechnique Fédérale de Lausanne (EPFL) \\ Signal Processing Institute \\ CH-1015 Lausanne, Switzerland \\ \{olivier.steiger,touradj.ebrahimi\}@epfl.ch
}

\section{Andrea Cavallaro}

\begin{abstract}
Perceptual prefiltering is the process of enhancing relevant portions of an image or of a video, and of simplifying contextual information in order to improve the perceived quality or the compression ratio. In this paper, we discuss the results of subjective quality evaluation experiments performed to assess the impact of perceptual prefiltering on video coding and we propose an objective quality metric that mimics the behavior of human observers. The predicted performance of the proposed metric is consistent with the subjective evaluation scores. Experimental results demonstrate that perceptual prefiltering leads to quality improvements by up to $10 \%$ at low bitrates.
\end{abstract}

\section{INTRODUCTION}

Perceptual prefiltering aims at mimicking the way humans treat visual information in order to improve the compression ratio of image and video coders. The overall image quality can be improved by degrading image areas that are not expected to attract the attention of a viewer in order to improve the quality (i.e. the associated bit allocation) of areas that observers are looking at [1]. To enable perceptual prefiltering, relevant portions of visual information (foreground) need to be separated from contextual information (background).

Previous work on perceptual prefiltering is based mainly on low-level features. Non-linear integration of low-level visual cues that mimics the processing in primate occipital and posterior parietal cortex is used in [2]. Visual cues are combined into a saliency map that modulates encoding priority. In [3], block importance is determined directly in the DCT domain by using a discontinuity height measure, which gives the contrast of dominant discontinuities within the block. Highly contrasted discontinuities are considered to be visually important. Other methods consider highlevel information (semantics) $[4,5]$. In [4], each frame frame is subdivided into a number of classes of relevance that are coded at a different level of quality by an object-based encoder. The definition of the classes depends on the task to be performed. For applications such as video conference or news broadcasting, faces may represent the classes to be considered, whereas in applications such as video surveillance and sport broadcasting, motion can be used for segmenting moving objects. In [5], each frame of the sequence is separated into foreground and background classes based on motion information. Then, after background simplification, both parts are re-composited together and coded by a framebased encoder (Fig. 1).

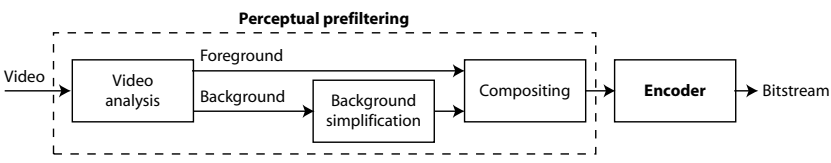

Fig. 1. Perceptual prefiltering based on semantic information is the process of video analysis, followed by background simplification and compositing.

In this paper, we quantify the impact of perceptual prefiltering with subjective experiments and show that background alterations resulting from perceptual prefiltering do not impair overall quality at low bitrates. Moreover, we propose an objective quality metric that mimics the behavior of human observers. The metric overcomes the limitations of subjective evaluation experiments that are expensive, time consuming and cannot be used to assess video quality in real time.

The paper is organized as follows. Subjective experiments are reported and discussed in Section 2. The objective quality metric is presented and validated in Section 3. Finally, we draw conclusions in Section 4.

\section{SUBJECTIVE EVALUATION}

\subsection{Experimental setup}

Four test sequences from the MPEG-4 Video Content Set are used for subjective performance evaluation: Children, Coastguard, Hall monitor and Akiyo. The sequences include deforming and rigid objects of different size, complex as well as simple background, and different types of motion. The TMPGEnc 2.521.58.169 MPEG-1 codec with constant bitrate (CBR) rate control is used. Coding bitrates are chosen so as to range from the lowest bitrate supported by the codec, up to perceptually lossless coding. Since we expect results to stabilize at high bitrates, tested rates are distributed exponentially: 200, 250, 300 and $500 \mathrm{Kbit} / \mathrm{s}$ for all sequences, plus $150 \mathrm{Kbit} / \mathrm{s}$ for Akiyo \& Hall monitor, and $100 \mathrm{Kbit} / \mathrm{s}$ for Akiyo.

Perceptual prefiltering is either achieved by lowpass filtering, or by replacing the original background by a static background shot (Hall monitor). The foreground is hand-segmented. The preprocessing methods under analysis are: (1) spatial resolution reduction; (2) perceptual prefiltering with lowpass filtering; (3) perceptual prefiltering with static background. In Fig. 2, a sample frames from each sequence coded with MPEG-1 at $150 \mathrm{Kbit} / \mathrm{s}$ using perceptual prefiltering with lowpass filtering is given. Clearly, 


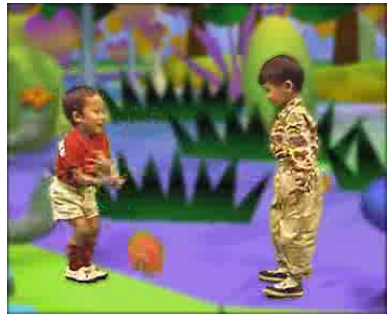

(a)

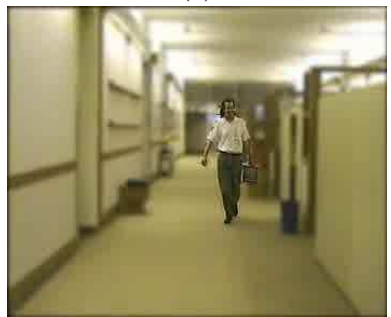

(c)

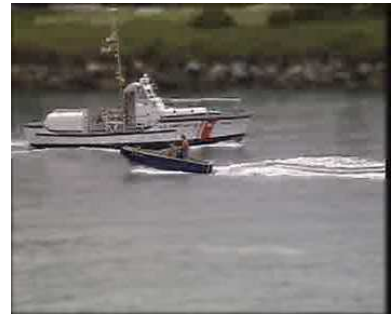

(b)

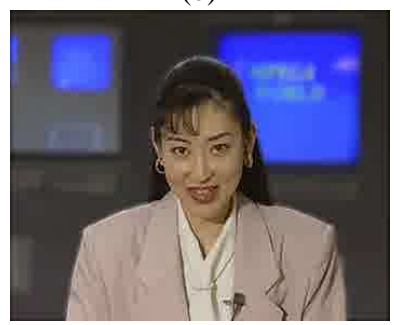

(d)
Fig. 2. Sample frame coded with MPEG-1 at $150 \mathrm{Kbit} / \mathrm{s}$ using perceptual prefiltering with lowpass filtering. (a) Children; (b) Coastguard; (c) Hall monitor; (d) Akiyo.

lowpass filtering of the background does not inhibit the main content message.

The conditions for subjective evaluation experiments follow the Absolute Category Rating (ACR) evaluation method, according to ITU-T Recommendation P.910 [6]. ACR is well-suited for qualification tests (i.e., to compare the performance of different coding strategies), as the method does not use explicit references. Twenty non-expert observers of different ages and backgrounds are presented a series of video sequences in random order; the presentation order is modified for each observer. Each observer participate to one sessions and each session contains 75 presentations. After each presentation, observers rate the quality of the sequence on a scale ranging from 0 (bad) to 100 (excellent). The presentation duration is 8 seconds and maximum 10 seconds are allowed for voting. Before each session, the range of qualities is presented to the observers in a training phase.

\subsection{Statistical analysis of subjective evaluation results}

Subjective experiments produce distributions of integer values, each number corresponding to one vote. These distributions exhibit a number of variations due to the difference in judgement between observers, and to the effect of a variety of conditions associated with the experiment. Specifically, a session consists of a number of presentations $L$. A presentation is obtained by applying one of a number of test conditions $J$, to one of a number of test sequences $K$. Each combination of test sequence and test condition may be repeated a number of times $R$. The mean score for each presentation, $\bar{u}_{j k r}$, is then given by

$$
\bar{u}_{j k r}=\frac{1}{N} \sum_{i=1}^{N} u_{i j k r},
$$

where $u_{i j k r}$ is the score of observer $i$ for test condition $j$, sequence $k$, and repetition $r . N$ is the total number of observers. The asso-
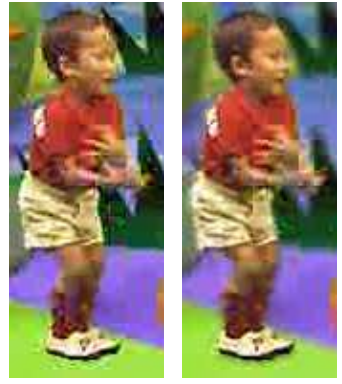

(a)
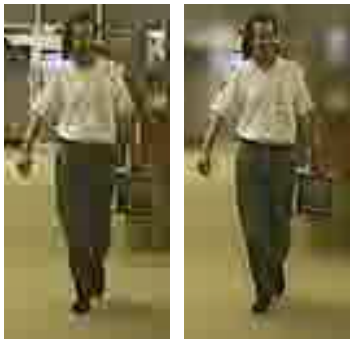

(c)
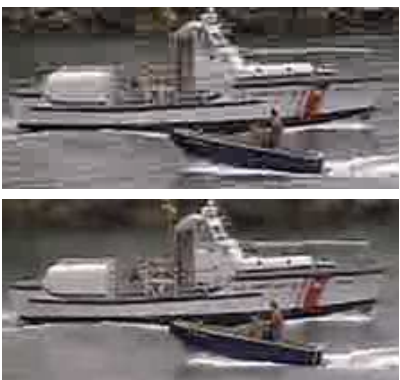

(b)

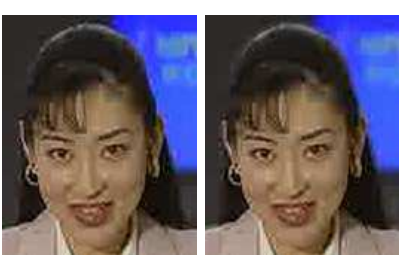

(d)
Fig. 3. Frame details with and without semantic prefiltering. (Left/top) coded original; (right/bottom) perceptual prefiltering with lowpass filtering. (a) Children; (b) Coastguard; (c) Hall monitor; (d) Akiyo.

ciated confidence interval is derived from the standard deviation and size of each sample. It is proposed to use the $95 \%$ confidence interval, which is given by $\left[\bar{u}_{j k r}-\delta_{j k r}, \bar{u}_{j k r}+\delta_{j k r}\right]$, where $\delta_{j k r}=1.96 \cdot\left(S_{j k r} / \sqrt{N}\right) . S_{j k r}$ is the standard deviation for each presentation.

Votes from unreliable observers are discarded aid of a screening procedure, organized in two stages. The first stage ensures that responses were entered accurately and in accordance with the experiment instructions. In the second stage, the variability of the data is reduced using the two-step method described in Annex 2 of ITU-R Recommendation BT.500-11 [7]. First, an expected range of values is calculated for each presentation. Then, the expected ranges are applied to the judgement of each observer. Finally, a subject is rejected for being erratic on both sides of the range, but not for being always above or always below the expected range. The results of subjective quality evaluation experiments are summarized in Figure 4. The graphs show the mean quality and associated $95 \%$ confidence interval as a function of coding bitrate.

\subsection{Discussion}

From Fig. 4, it is possible to notice that perceptual prefiltering (23) has a positive impact at low bitrates, in particular when the background is replaced by a frame or a sprite representing the background (3) (Hall monitor). At bitrates up to $300 \mathrm{Kbit} / \mathrm{s}$, this increases the mean quality by up to 10 points as compared to the coded original. This is because inter-coded, static background blocks do not produce residue, so most of the available bitrate can be allocated to foreground objects.

Lowpass-filtering (2) has a lesser impact. Viewers notice the improvement of foreground quality due to additional bandwidth 


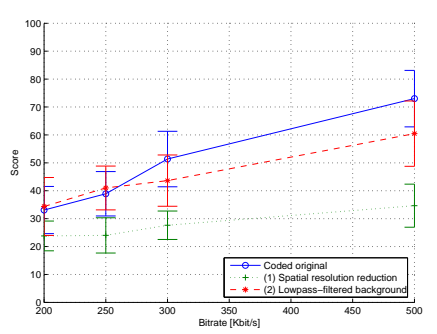

(a)

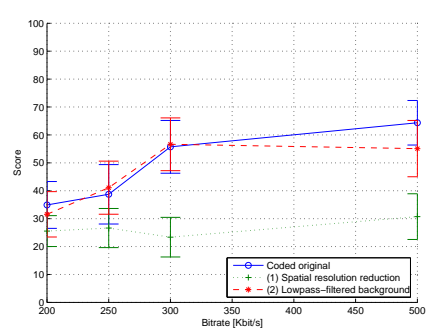

(b)

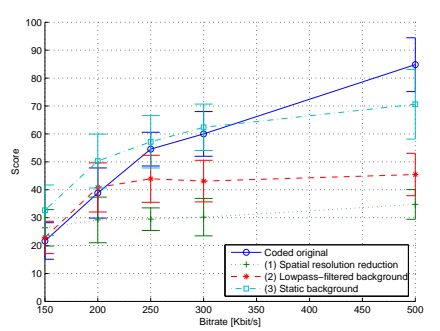

(c)

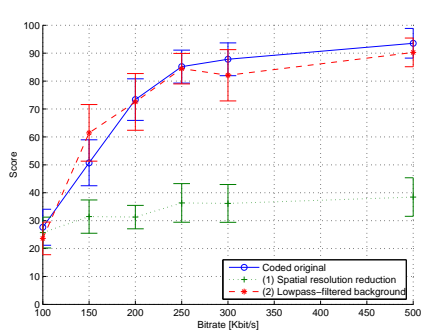

(d)

Fig. 4. Subjective evaluation results of perceptually prefiltered video. The graphs show the mean quality and associated $95 \%$ confidence interval as a function of bitrate. (a) Children; (b) Coastguard; (c) Hall monitor; (d) Akiyo.

freed by the filter, but at the same time they are annoyed by the loss of background information. For Akiyo, the quality of lowpassfiltered and coded original versions is similar over the entire bitrate range. This is because the background of the original sequence is out of focus, and thus has few high-frequency components. For Hall monitor, the mean quality of lowpass-filtering is slightly above that of the coded original $(+1.5)$ at bitrates up to 200 $\mathrm{Kbit} / \mathrm{s}$. The same is true $(+1.3)$ for Children at bitrates up to 250 Kbit/s. For Coastguard, lowpass-filtering has been rated above the coded original $(+2.5)$ at bitrates of 250 and $300 \mathrm{Kbit} / \mathrm{s}$, but below $(-3.5)$ at the lowest bitrate of $200 \mathrm{Kbit} / \mathrm{s}$. This is because at 200 $\mathrm{Kbit} / \mathrm{s}$, foreground objects are corrupted by heavy artifacts in both versions, whereas at 250 and $300 \mathrm{Kbit} / \mathrm{s}$, lowpass-filtering notably reduces artifacts that are still visible in the coded original. The improvement of foreground quality can be verified in Fig. 3. Semantic prefiltering notably enhances the face in Children and the boats in Coastguard.

Background simplifications resulting from perceptual prefiltering (2-3) do not penalize overall quality at low bitrates (100-250 $\mathrm{Kbit} / \mathrm{s})$. In fact, image degradations are strong at such bitrates, and improvements on important image parts due to the additional bandwidth freed by background simplification are positively perceived. At high bitrates on the other hand, both foreground and background are coded at high quality. Thus, background alterations are easily noticed by observers and degrade the overall impression.

\section{OBJECTIVE EVALUATION}

\subsection{Quality metric}

Subjective evaluation experiments are expensive, time consuming and cannot be used to assess video quality in real time. An objective evaluation metric would therefore be desirable. An objective video distortion measure that emulates human judgement needs to account for different image areas and their relevance to the observer. This aspect can be considered with the traditional Mean Squared Error (MSE) by weighting different image areas according to their semantics. This leads to the semantic mean squared error, SMSE, defined:

$$
\mathrm{SMSE}=\sum_{k=1}^{N} \frac{w_{k}}{\left|C_{k}\right|} \sum_{(i, j) \in C_{k}} d^{2}(i, j),
$$

where $N$ is the number of classes and $w_{k}$ the weight of class $k$. Class weights are chosen depending on the semantics, with $w_{k} \geq 0, \forall k=1, \ldots, N$ and $\sum_{i=1}^{N} w_{k}=1 . C_{k}$ is the set of pixels belonging to the object class $k$, and $\left|C_{k}\right|$ is its cardinality. The error $d(i, j)$ between the original image $I_{O}$ and the distorted image $I_{D}$ in Eq. (2) is the pixel-wise color distance. The color distance is computed in the 1976 CIE Lab color space in order to consider perceptually uniform color distances with the Euclidean norm. The final quality evaluation metric, the semantic peak signal-to-noise ratio SPSNR, uses SMSE instead of MSE as compared to PSNR. When the classes are foreground and background, then $N=2$ in Eq. (2), and $w_{f}$ is the foreground weight. The background weight is thus $\left(1-w_{f}\right)$. The value of $w_{f}$ is computed as described in the following section.

\subsection{Foreground relevance}

Subjective experiments quantify the amount of attention that we pay to the foreground and to the background. The foreground weight, $w_{f}$, is determined by minimizing the Pearson correlation [8] between SPSNR and subjective results. For the sequence Akiyo, where the foreground covers a large area and the background is simple, the observers focused mostly on foreground, thus leading to a value of $w_{f}=0.97$. For Hall monitor, whose background is more complex and objects are smaller, the foreground attracted slightly more the attention than the background $\left(w_{f}=0.55\right)$. The sequence Children has a very complex and colored background that attracted the observer's attention, thus resulting in foreground and background being equally weighted $\left(w_{f}=0.5\right)$. The sequence Coastguard contains camera motion. This prevented the observer from focusing on background steadily, even though it is quite complex. In this case, $w_{f}=0.7$. In general, results confirm that large moving objects and complex background tend to attract users attention.

Based on the data collected with subjective experiments, we predict the foreground weight based on the following formula:

$$
w_{f}=\left(\alpha-\beta \cdot \sigma_{b}\right) \cdot r+\gamma \cdot v+\left(\sigma_{b}+1\right) \cdot \delta,
$$

where $r$ represents the portion of the image occupied by foreground pixels: $r=\left|C_{f}\right| /\left(\left|C_{f}\right|+\left|C_{b}\right|\right)$, with $\left|C_{f}\right|$ and $\left|C_{b}\right|$ representing the number of foreground and background pixels, respectively. The background complexity is taken into account with $\sigma_{b}$, the standard deviation of the luminance of background pixels. The presence of camera motion is considered with $v: v=1$ for moving camera, and $v=0$ otherwise. $\alpha, \beta, \gamma$ and $\delta$ are constants whose values have been determined based on the results of the subjective experiments: $\alpha=5.7, \beta=0.108, \gamma=0.2$ and $\delta=0.01$. 


\subsection{Discussion}

In Fig. 5(a), Eq. 3 has been used to calculate the foreground weight, $w_{f}$, as a function of time for Hall monitor. The graphs is subdivided into four zones. In zone 1 (frames 1-23), a person enters the room from the left. In zone 2 (frames 24-80), the person walks away from the camera, and a second person enters the room from the right. In zone 3 (frames 81-248), the person on the right walks to the camera, and the person on the left walks away from the camera and leaves the room. In zone 4 (frames 249-293), the person on the right continues to walk towards the camera. The foreground weight, $w_{f}$, increases when the portion of the image occupied by foreground pixels, $r$, increases as well. This reflects the fact that large moving objects tend to attract the attention. For instance, the average foreground weight in zone 2, where only one person is visible, is $w_{f}=0.548$, whereas $w_{f}=0.55$ in zone 3 , with two persons.

In Fig. 5(b), SPSNR is plotted as a function of time for Hall monitor. The average quality improvement obtained by semantic prefiltering with static background (3) as compared to the coded original is $1.84 \mathrm{~dB}$. This reflects the improvement noticed by human observers in subjective experiments. The average quality with lowpass-filtered background (2) is inferior to the coded original by $1.38 \mathrm{~dB}$. Note that between frames 1 and 11, the performances of coded original (1) and semantic prefiltering with static background (3) are similar, because a very limited portion of the image is occupied by the foreground.

\section{CONCLUSIONS}

The effectiveness of perceptual prefiltering in improving the quality at low bitrates has been quantified and analyzed subjectively and objectively. Moreover, an objective video distortion measure that emulates human judgement has been described that accounts for different image areas and their relevance to the observer. Subjective experiments have confirmed that large moving objects and complex background tend to attract observer's attention. At low bitrates, perceptual prefiltering improves quality by up to $10 \%$. In particular, the replacement of the background with a still background shot results in significantly more bandwidth being allocated to important image regions, without degrading the overall quality. This is very important for applications with fixed cameras, such as news broadcast and video surveillance, or when it is possible to compute a sprite of the background, such as sport broadcasting.

\section{REFERENCES}

[1] A. P. Bradley and F. W. M. Stentiford, "Visual attention for region of interest coding in JPEG 2000," Journal of Visual Communication and Image Representation, vol. 14, pp. 232250, 2003.

[2] L. Itti, "Automatic foveation for video compression using a neurobiological model of visual attention," IEEE Trans. on Image Processing, vol. 13, no. 10, pp. 1304-1318, 2004.

[3] TaeYong Kim and Jong Soo Choi, "Content-based video transcoding in compressed domain," Signal Processing: Image Communication, vol. 17, pp. 497-507, 2002.

[4] Rita Cucchiara, Costantino Grana, and Andrea Prati, "Semantic transcoding for live video server," in Proc. of Tenth ACM Int. Conf. on Multimedia, 2002, pp. 223-226.

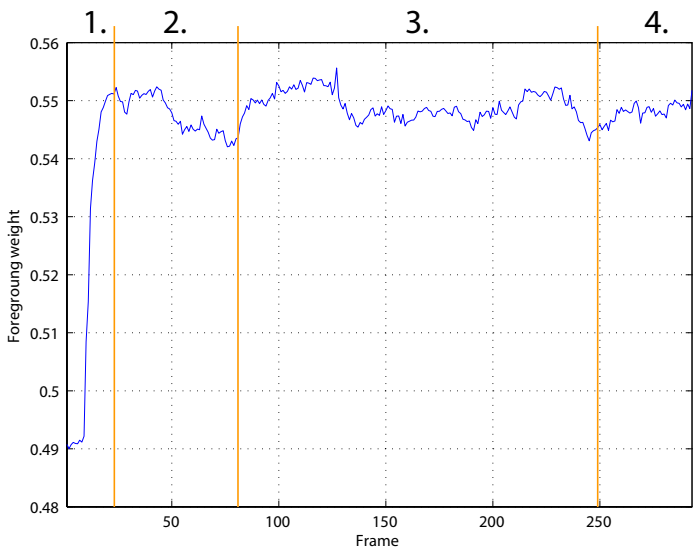

(a)

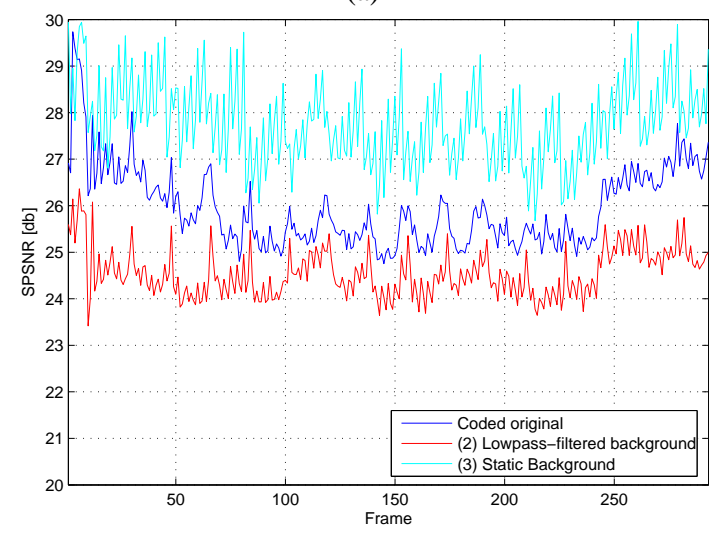

(b)

Fig. 5. Objective evaluation results for Hall monitor. (a) Foreground weight, $w_{f}$, as a function of time; (b) SPSNR as a function of time.

[5] Andrea Cavallaro, Olivier Steiger, and Touradj Ebrahimi, "Perceptual prefiltering for video coding," in Proc. of IEEE Int. Symposium on Intelligent Multimedia, Video and Speech Processing, ISIMP'04, October 2004.

[6] ITU, "Subjective video quality assessment methods for multimedia applications," Tech. Rep. P.910, ITU-T Recommandation, September 1999.

[7] ITU, "Methodology for the subjective assessment of the quality of television pictures," Tech. Rep. BT.500-11, ITU-R Recommandation, 2002.

[8] David Freedman, Robert Pisani, and Roger Purves, Statistics, W.W. Norton \& Company, 3 edition, 1997. 